\title{
Composición Factorial de una Escala Autoeficacia en Conductas de Cuidado de la Salud en Estudiantes de Ingeniería
}

\author{
Francisco J. Flores, Jesús E. Peinado, Martha Ornelas y Lepoldo López \\ Universidad Autónoma de Chihuahua. Facultad de Educación Física y Ciencias del Deporte. \\ DES Salud CAs 101 y 121. Calle Escorza 900, CP 31000 Chihuahua, Chih.- México. \\ (e-mail:fflores@uach.mx,.jpeinad@uach.mx,mornelas@uach.mx y llopez@uach.mx)
}

Recibido Ene. 18, 2012; Aceptado Feb. 15, 2012; Versión final recibida Feb. 17, 2012

\begin{abstract}
Resumen
El presente estudio analiza las propiedades sicométricas de la Escala Autoeficacia en Conductas de Cuidado de la Salud Física. La muestra total fue de 592 sujetos; 143 mujeres y 449 hombres, alumnos de primer ingreso a las licenciaturas de ingeniería que se ofrecen en la Universidad Autónoma de Chihuahua en México, con una edad media de 18.16 años. La estructura factorial del cuestionario se analizó a través de un análisis factorial confirmatorio. El análisis ha mostrado que una estructura tetrafactorial es viable y adecuada de acuerdo a los requisitos sicométricos establecidos. La estructura de cuatro factores, atendiendo a criterios estadísticos ha mostrado adecuados indicadores de ajuste, fiabilidad y validez. Los resultados de los análisis factoriales llevados a cabo con las submuestras 1 y 2 , indican la existencia de fuertes evidencias de la estabilidad de la estructura factorial.
\end{abstract}

\section{Factor Structure of a Self-Efficacy Questionnaire in Health Care Behavior in Engineering Students}

\begin{abstract}
The present study analyses the psychometric properties of a self-efficacy questionnaire behavior of health care. The total sample comprised 592 participants; 143 women and 449 men, all of them freshmen at Universidad Autónoma de Chihuahua in Mexico in the area of engineering, with a mean age of 18.16 years. Psychometric analysis showed that a four-factorial structure is feasible and adequate according to the established psychometric requirements. The factor structure of the questionnaire was analyzed using confirmatory factor analysis. The structure of the four factors, following statistical criteria, showed adequate fitting indicators, reliability and validity. In addition, factor analysis conducted with subsamples 1 and 2 indicated the presence of strong evidence of factor structure stability.
\end{abstract}

Keywords: self-efficacy, factor structure, measurement invariance, factor analysis 


\section{INTRODUCCIÓN}

La propia motivación impulsa al ser humano a emprender conductas específicas en función de los logros que éste pretende alcanzar. Más no basta conocer con claridad aquello que deseamos lograr, ni tampoco el mejor medio para poder conseguirlo. Tampoco, basta con ser capaz de; es preciso juzgarse capaz de utilizar las capacidades y las habilidades personales ante circunstancias muy diversas. La percepción de las personas acerca de su propia eficacia se alza como un requisito fundamental para desarrollar con éxito las acciones conducentes al logro de los objetivos personales (Ornelas et al., en prensa; Vera et al., 2011). Dicha autopercepción, denominada autoeficacia, ejerce una profunda influencia en la elección de tareas y actividades, en el esfuerzo y en la perseverancia de las personas cuando se enfrentan a determinados retos e incluso en las reacciones emocionales que experimentan ante situaciones difíciles (León-Rubio et al., 2011; Pérez et al., 2011; Wolters, 2004). En definitiva, las creencias de autoeficacia representan un mecanismo cognitivo que media entre el conocimiento y la acción y que determina, junto con otras variables, el éxito de las propias acciones (Castañeda et al., 2010; Carbonero y Merino, 2008; Pérez et al., 2011; Sansinenea et al., 2008). Las investigaciones revelan también, que altos niveles de autoeficacia tienen consecuencias beneficiosas para el funcionamiento del individuo y su bienestar general (Klein-Hessling et al., 2005; Ornelas et al., 2011).

De acuerdo a las formulaciones de Bandura (1992, 1997) cuando un individuo se plantea la posibilidad de llevar a cabo una conducta de salud o cambiar un hábito no saludable hay cuatro elementos que explican la decisión resultante: 1) la creencia de que una situación determinada es perjudicial; 2) la creencia de que un cambio de comportamiento puede reducir la supuesta amenaza y 3) la creencia de que es suficientemente competente como para adoptar una conducta beneficiosa o para dejar de practicar una que resultaría dañina. Es este último aspecto al que alude el concepto de expectativas de autoeficacia: las creencias acerca de la capacidad para ejercer control sobre la propia conducta y el medio en el que ésta tiene lugar.

Desde la Teoría del Aprendizaje Social de Bandura se asume entonces que las expectativas de autoeficacia son un importante predictor de las intenciones y acciones de los individuos frente a diversas situaciones; ya que un elevado nivel de autoeficacia percibida se ha mostrado como un elemento protector que hace aumentar la motivación, disminuir las alteraciones emocionales, al tiempo que mejora las conductas saludables en el cuidado físico. De hecho, frente a lo difícil que puede ser el motivar a la adopción de conductas que promuevan la salud o el detener conductas nocivas para ésta, la autoeficacia ha mostrado consistentemente ser un factor de gran importancia (Olivari y Urra, 2007).

La autoeficacia percibida pues, desempeña un papel fundamental en el funcionamiento humano puesto que afecta el comportamiento no sólo de manera directa, sino también por su impacto en otros determinantes claves tales como metas y aspiraciones, expectativas de resultados, tendencias afectivas y percepción de los impedimentos y oportunidades que se presentan en el medio social (Bandura, 1997). Las creencias de autoeficacia influyen en los pensamientos de las personas, en los cursos de acción que ellas eligen para perseguir; los desafíos y metas que ellas se plantean para sí mismas y su compromiso con los mismos; la cantidad de esfuerzo que invierten en determinadas tareas; los resultados que esperan alcanzar por sus esfuerzos; la magnitud de su perseverancia frente a los obstáculos; su resistencia a la adversidad; el nivel de estrés y depresión que experimentan cuando se enfrentan con demandas exigentes del ambiente y los logros que alcanzan.

En este trabajo se analiza la consistencia interna y la estructura factorial de un instrumento de autoreporte que permite identificar conductas de cuidado de la salud, cuyo nivel de autoeficacia percibido por los alumnos de nuevo ingreso representen un área de oportunidad o mejora; en relación con el resto de los alumnos, aportando evidencias y datos que propicien la intervención educativa dentro de una perspectiva de atención a la diversidad en el aula. 
En el presente estudio interesa pues, no solo la estructura factorial del instrumento, sino también la equivalencia psicométrica del mismo en distintos grupos; ya que en el contexto de la comparación intergrupal, es indispensable plantearse la necesidad de llevar a cabo la adaptación de un instrumento de medida psicológica que cumpla con todos los criterios de equivalencia, pero sobre todo plantearse si la misma estructura factorial es aplicable a distintos grupos de sujetos o, de modo más genérico, a distintas poblaciones (Abalo et al., 2006)

\section{METODOLOGÍA}

\section{Sujetos}

La muestra de 592 sujetos, $143(24.2 \%)$ mujeres y 449 (75.8\%) hombres se obtuvo mediante un muestreo por conveniencia, tratando de abarcar la representatividad de las diferentes licenciaturas de ingeniería que se ofrecen en la Universidad Autónoma de Chihuahua; luego, la muestra, fue aleatoriamente dividida en dos submuestras $(50 \%$ y $50 \%)$ utilizando el Statistical Package for the Social Sciences (SPSS) en su versión 17.0; con el fin de realizar estudios paralelos que permitieran corroborar y verificar los resultados obtenidos (validación cruzada).

La primera mitad (submuestra 1) quedo constituida por 315 sujetos; 74 (23.5\%) mujeres y 241 $(76.5 \%)$ hombres. Las edades fluctúan entre los 17 y 20 años, con una media de 18.15 y una desviación estándar de 0.72 años. La segunda mitad (submuestra 2) quedo compuesta por 277 sujetos; $69(24.9 \%)$ mujeres y $208(75.1 \%)$ hombres. Las edades fluctúan entre los 17 y 20 años, con una media de 18.17 y una desviación estándar de 0.73 años.

\section{Instrumento}

Escala Autoeficacia en Conductas de Cuidado de la Salud Física (EACCSF), encuesta tipo Likert, asistida por computadora, de 8 ítems (donde, la escala de medición de los ítems 7 y 8 se invierte para que concuerde con la de los otros 6 ítems) relacionados con conductas de cuidado de la salud; donde el encuestado responde, en una escala de 0 a 10 , la frecuencia con que actualmente, en forma ideal y si se esfuerza en cambiar, realizaría o manifestaría una acción (Blanco et al., 2011). Se eligió este tipo de encuesta por ser fácil de construir y de aplicar; además, de proporcionar una buena base para una primera ordenación de los individuos en la característica que se mide; en nuestro caso autoeficacia (Nunnally y Bernstein, 1995; Raviolo et al., 2010).

\section{Procedimiento}

Se invitó a participar en el estudio a los alumnos de primer ingreso de las licenciaturas de ingeniería que se ofrecen en la Universidad Autónoma de Chihuahua; los que aceptaron participar firmaron la carta de aceptación correspondiente. Luego se aplicó el instrumento antes descrito por medio de una computadora personal y utilizando el módulo administrador del instrumento del editor de escalas versión 2.0 (Blanco et al., 2007), en una sesión de aproximadamente 20 minutos; en los laboratorios o centros de cómputo de las unidades académicas participantes. Al inicio de cada sesión se hizo una pequeña introducción sobre la importancia de la investigación y de cómo acceder al instrumento. Las instrucciones de cómo responder se encontraban en las primeras pantallas; antes del primer reactivo del instrumento. Al término de la sesión se les agradeció su participación.

Una vez aplicado el instrumento se procedió a recopilar los resultados por medio del módulo generador de resultados del editor de escalas versión 2.0 (Blanco et al., 2007).

\section{Análisis de datos}

Con el fin de obtener una prueba que presente las mejores propiedades para la conformación de los puntajes de autoeficacia en conductas de cuidado de la salud en universitarios de ingeniería, el 
análisis psicométrico se realizó en dos etapas: 1) análisis factorial exploratorio y 2) análisis factorial confirmatorio y de invarianza factorial.

Para determinar el número mínimo de factores comunes capaces de reproducir, de un modo satisfactorio, las correlaciones observadas entre los ítems del instrumento, se realizaron sendos análisis factoriales exploratorios con las submuestras 1 y 2 , a partir del método de factores principales, tomando como base el criterio de Kaiser-Guttman (Costello y Osborne, 2005) y que la comunalidad, o proporción de la varianza explicada por la solución factorial para cada ítem debe ser mayor o igual a .50; después de una rotación varimax (Costello y Osborne, 2005). Luego mediante el coeficiente $\alpha$ de cronbach se estimó la consistencia interna para cada factor retenido como una medida de su fiabilidad (Nunnally y Bernstein, 1995; Elosua y Zumbo, 2008).

Para conducir el análisis factorial confirmatorio en la submuestra 2, se utilizó el software AMOS 16 (Arbuckle, 2007), las varianzas de los términos de error fueron especificados como parámetros libres, en cada variable latente (factor) se fijó uno de los coeficientes estructurales asociados a uno, para que su escala sea igual a la de una de las variables superficiales (ítems). El método de estimación empleado fue el de Máxima Verosimilitud; siguiendo la recomendación de Thompson (2004), en el sentido de que cuando se emplea análisis factorial confirmatorio se debe corroborar no sólo el ajuste de un modelo teórico sino que es recomendable comparar los índices de ajuste de varios modelos alternativos para seleccionar el mejor.

Para evaluar el ajuste del modelo se emplearon el estadístico Chi-cuadrado, el índice de bondad de ajuste (GFI), el residuo cuadrático medio (RMR), el error cuadrático medio de aproximación (RMSEA) y el índice de validación cruzada esperada (ECVI) como medidas absolutas de ajuste. El índice de bondad ajustado (AGFI), el Índice Tucker-Lewis (TLI), el Índice del ajuste normal (NFI) y el índice de bondad de ajuste comparativo (CFI) como medidas de ajuste incremental. El índice de ajuste normado de parsimonia (PNFI), el índice de calidad de ajuste de parsimonia (PGFI), la razón de Chi-cuadrado sobre los grados de libertad (CMIN/GL) y el Criterio de Información de Akaike (AIC) como medidas de ajuste de parsimonia (Gelabert et al., 2011; Leighton et al., 2007).

Para comprobar la estructura factorial de la escala obtenida a partir de la submuestra 2 (análisis factorial confirmatorio, primera solución factorial), se realizó un segundo análisis factorial confirmatorio en la submuestra 1.

\section{RESULTADOS}

\section{Análisis factorial exploratorio}

Para determinar el número mínimo de factores comunes capaces de reproducir, de un modo satisfactorio, las correlaciones observadas entre los 8 ítems del instrumento empleado, se realizó, en la submuestra 1, la extracción de éstos a partir del método de componentes principales. La significación del Test de Barlett (505.919; p<.0001) y el KMO (.675) mostraron una adecuada correlación entre los ítems y una buena adecuación muestral respectivamente. Detectándose, después de una rotación varimax, una estructura de cuatro factores; el conjunto de los factores seleccionados explicaron el $75.53 \%$ de la varianza (Tablas 1 y 2 ).

Con el fin validar la solución factorial obtenida con la submuestra 1 , se realizó nuevamente un análisis de factores principales con los datos de la submuestra 2; encontrándose de nuevo una estructura de cuatro factores. El conjunto de los cuatro factores explicaron el $76.42 \%$ de la varianza (Tablas 1 y 2). La significación del Test de Barlett $(507.360 ; p<.0001)$ y el KMO (.714) muestran nuevamente, una adecuada correlación entre los ítems y una buena adecuación muestral respectivamente. 
Tabla 1: Autovalores y porcentaje de varianza explicada por los factores retenidos. Análisis factorial exploratorio Submuestras 1 y 2 . Soluciones rotadas.

\begin{tabular}{lcccccc}
\hline & \multicolumn{3}{c}{ Submuestra 1 } & \multicolumn{3}{c}{ Submuestra 2 } \\
\cline { 2 - 7 } \multicolumn{1}{c}{ Factor } & Autovalor $\%$ varianza \% acumulado & Autovalor & \% varianza \% acumulado \\
\hline 1. Alimentación & 1.71 & 21.42 & 21.42 & 1.79 & 22.43 & 22.43 \\
2. Adicciones & 1.55 & 19.34 & 40.76 & 1.48 & 18.47 & 40.90 \\
3. Salud Física & 1.49 & 18.61 & 59.37 & 1.65 & 20.62 & 61.52 \\
4. Hidratación & 1.29 & 16.16 & 75.53 & 1.19 & 14.90 & 76.42 \\
\hline
\end{tabular}

Tabla 2: Ítems agrupados por factor. Análisis factorial exploratorio Submuestras 1 y 2 . Soluciones rotadas.

\begin{tabular}{|c|c|c|c|c|c|c|c|c|c|}
\hline & \multirow{2}{*}{ Ítem } & \multicolumn{4}{|c|}{ Submuestra 1} & \multicolumn{4}{|c|}{ Submuestra 2} \\
\hline & & 1 & 2 & 3 & 4 & 1 & 2 & 3 & 4 \\
\hline 4 & Tengo horarios fijos para mis comidas & .87 & & & & .86 & & & \\
\hline 2 & Realizo tres comidas al día & .85 & & & & .88 & & & \\
\hline 8 & Consumo bebidas alcohólicas & & .87 & & & & .84 & & \\
\hline 7 & Consumo tábaco & & .86 & & & & .84 & & \\
\hline 1 & Me cuido bien físicamente & & & .85 & & & & .80 & \\
\hline 3 & Realizo ejercicio físico durante ... & & & .80 & & & & .89 & \\
\hline 5 & Bebo más de 6 vasos de agua al día & & & & .92 & & & & .95 \\
\hline 6 & Consumo 2 o más piezas de fruta al día & & & & .58 & & & & .47 \\
\hline
\end{tabular}

Fiabilidad de las subescalas (consistencia interna)

Las subescalas (factores) resultantes en los análisis factoriales exploratorios, de ambas submuestras, poseen alfas cercano a .7, evidenciando una consistencia interna adecuada para este tipo de subescalas, particularmente si se considera el número reducido de ítems (Tabla 3).

Tabla 3: Coeficientes de consistencia interna de los factores obtenidos en los análisis factoriales exploratorios submuestras 1 y 2.

\begin{tabular}{lcc}
\hline \multicolumn{1}{c}{ Factor } & $\alpha$ Submuestra 1 & $\alpha$ Submuestra 2 \\
\hline 1. Alimentación & .718 & .757 \\
2. Adicciones & .676 & .689 \\
3. Salud Física & .684 & .692 \\
4. Hidratación & .671 & .681 \\
\hline
\end{tabular}

\section{Análisis factorial confirmatorio}

Ahora, mediante el análisis factorial confirmatorio sobre los datos de la submuestra 2, se contrasta la solución factorial resultante del análisis factorial exploratorio realizado con los datos de la submuestra 1; con el fin de obtener modelos congenéricos y, al mismo tiempo, probar la validez de constructo de las dimensiones y variables estudiadas.

De acuerdo a los resultados de la Tabla 4 el análisis factorial confirmatorio de 8 ítems agrupados en cuatro factores es óptimo (GFI .986 y RMSEA .039) y de acuerdo a las medidas de ajuste 
incremental y de parsimonia (Tablas 5 y 6 ) significativamente superior al modelo independiente y muy similar al modelo saturado.

Tabla 4 Medidas absolutas de ajuste para los modelos generados. Primer y segundo análisis factorial confirmatorio submuestras 1 y $2 ;{ }^{*} p<.01$.

\begin{tabular}{lccccc}
\hline & \multicolumn{5}{c}{ Índice de Ajuste } \\
\cline { 2 - 5 } Modelo & $\chi^{2}$ & GFI & RMR & RMSEA & ECVI \\
\cline { 2 - 6 } Independiente & Primera solución factorial (submuestra 2) \\
Saturado & $513.877^{*}$ & .625 & 2.151 & .251 & 1.920 \\
4 factores & 0 & 1 & 0 & & 0.261 \\
& 17.180 & .986 & 0.288 & .029 & 0.222 \\
Independiente & Segunda solución factorial (submuestra 1) & \\
Saturado & $511.622^{*}$ & .670 & 1.888 & .235 & 1.680 \\
4 factores & 0 & 1 & 0 & & 0.229 \\
\hline
\end{tabular}

Tabla 5 Medidas de ajuste incremental para los modelos generados. Primer y segundo análisis factorial confirmatorio submuestras 1 y 2.

\begin{tabular}{lcccc}
\hline \multirow{2}{*}{ Modelo } & \multicolumn{4}{c}{ Índice de Ajuste } \\
\cline { 2 - 4 } & AGFI & \multicolumn{1}{c}{ TLI } & NFI & CFI \\
Independiente & .518 & 0 & 0 & 0 \\
Saturado & & & 1 & 1 \\
4 factores & .963 & .987 & .967 & .993 \\
\hline & Segunda solución factorial (submuestra 1) & \\
Independiente & .576 & 0 & 0 & 0 \\
Saturado & & & 1 & 1 \\
4 factores & .923 & .909 & .930 & .955 \\
\hline
\end{tabular}

Tabla 6 Medidas de ajuste de parsimonia para los modelos generados. Primer y segundo análisis factorial confirmatorio submuestras 1 y 2 .

\begin{tabular}{lcccc}
\hline \multirow{2}{*}{ Modelo } & \multicolumn{4}{c}{ Índice de Ajuste } \\
\cline { 2 - 5 } Independiente & Primera solución factorial & (submuestra 2) & AIC \\
Saturado & 0 & .486 & 18.353 & 529.877 \\
4 factores & 0 & & & 72.000 \\
\hline & .483 & .383 & 1.227 & 61.180 \\
Independiente & Segunda solución factorial (submuestra 1) \\
Saturado & 0 & .521 & 18.272 & 79.980 \\
4 factores & 0 & & & 72.000 \\
& .465 & .377 & 2.570 & 79.980 \\
\hline
\end{tabular}


Para comprobar la estructura factorial de la escala autoeficacia percibida en conductas de cuidado de la salud obtenida a partir de la submuestra 2 (análisis factorial confirmatorio, primera solución factorial), se realizó un segundo análisis factorial confirmatorio en la submuestra 1. La estructura factorial analizada plantea un modelo de cuatro factores, con adscripciones de los ítems basados en análisis factorial confirmatorio de la submuestra 2 (4 factores 8 ítems). De acuerdo a los resultados de la Tabla 4, el segundo análisis factorial confirmatorio (submuestra 1) indica que el modelo de medición de cuatro factores es aceptable (GFI .970 y RMSEA .071) y de acuerdo a las medidas de ajuste incremental y de parsimonia (ver Tablas 5 y 6 ) significativamente superior al modelo independiente y muy similar al modelo saturado.

En la figura 1 se presenta el modelo de medición del primer análisis factorial confirmatorio para los 8 ítems agrupados en cuatro factores, incluyendo los coeficientes de regresión estandarizados entre ítems y factores y las saturaciones factoriales estandarizadas (comunalidades) de cada uno de los ítems.

En los factores Alimentación y Salud Física todos los ítems resultan bien explicados a partir del constructo hipotetizado; con saturaciones factoriales estandarizadas por encima de .40. Mientras que los factores Adicciones e Hidratación presentan una saturación factorial baja en uno de sus ítems (8 y 5 , respectivamente).

Por su parte, todos los factores correlacionan entre sí de forma estadísticamente significativa $(p<.05)$. Las correlaciones entre Adicciones y los factores Alimentación, Salud Física e Hidratación pueden considerarse bajas, con valores de $.13, .23$ y .24 respectivamente, mientras que la relación entre estos tres últimos factores es alta; lo cual muestra que a medida que aumenta la autoeficacia percibida en alguno de los factores, también aumenta en los otros tres.

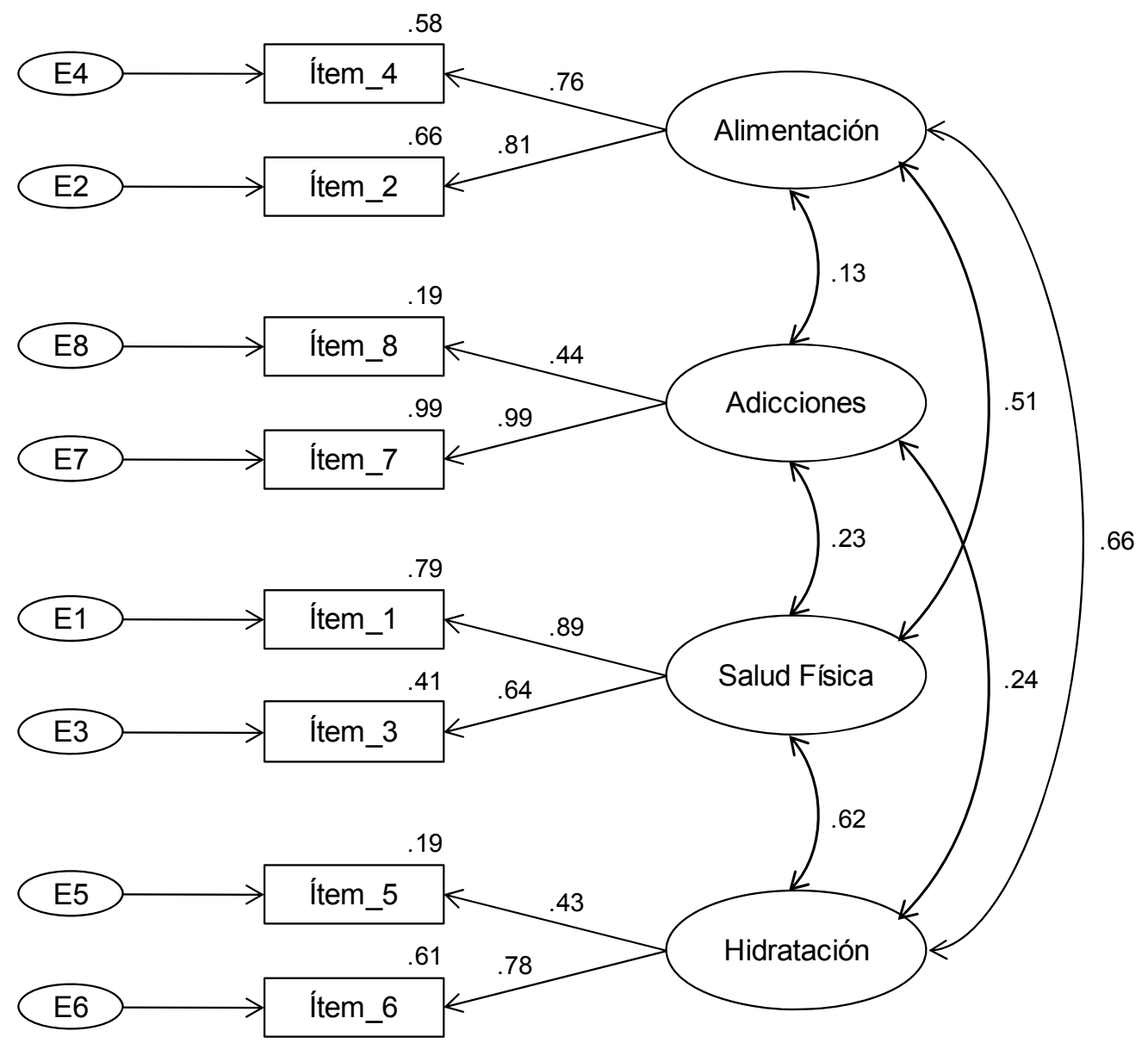

Fig. 1: Modelo de medición para la escala. Análisis factorial confirmatorio submuestra 2. Fuente: de los autores. 
En la figura 2 se presenta el modelo de medición del segundo análisis factorial confirmatorio para los 8 ítems agrupados en cuatro factores, incluyendo los coeficientes de regresión estandarizados entre ítems y factores y las saturaciones factoriales estandarizadas (comunalidades) de cada uno de los ítems.

De nuevo, en los factores Alimentación y Salud Física todos los ítems resultan bien explicados a partir del constructo hipotetizado; con saturaciones factoriales estandarizadas por encima de .40. Mientras que los factores Adicciones e Hidratación presentan una saturación factorial moderada en uno de sus ítems (8 y 5 , respectivamente).

Por su parte, todos los factores correlacionan entre sí de forma estadísticamente significativa $(p<.05)$. Las correlaciones entre Adicciones y los factores Alimentación, Salud Física e Hidratación pueden considerarse bajas, con valores de .10, .17 y .11 respectivamente, mientras que la relación entre estos tres últimos factores es alta; lo cual muestra que a medida que aumenta la autoeficacia percibida en alguno de los factores, también aumenta en los otros tres.

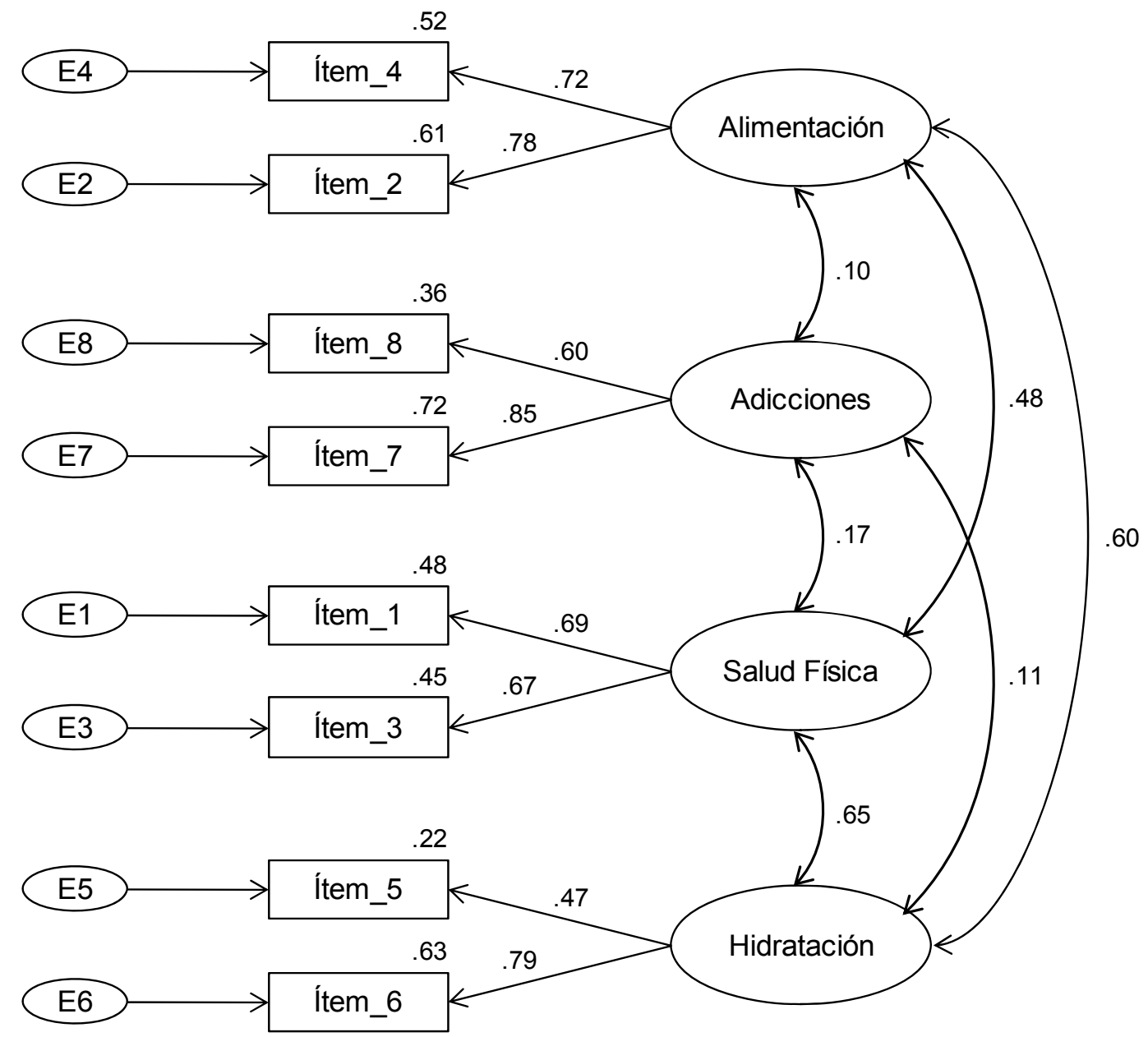

Fig. 2: Modelo de medición para la escala. Análisis factorial confirmatorio submuestra 1. Fuente: de los autores.

Invarianza de la estructura factorial en las dos submuestras

Para analizar la invarianza factorial del cuestionario se siguieron las recomendaciones de Abalo et al. (2006) estimando el mismo modelo en ambas muestras; los índices de ajuste obtenidos (Tabla 7) permiten aceptar la equivalencia de los modelos de medida básicos entre las dos muestras. Aunque el valor de Chi-cuadrado excede al exigido para aceptar la hipótesis de invarianza, el resto de índices contradicen esta conclusión (GFI .977; CFI .974; RMSEA .039; AIC 141.070) lo que nos permite aceptar el modelo base de la invarianza (modelo sin restricciones). 
Añadiendo al modelo base restricciones sobre las cargas factoriales caracterizamos la invarianza métrica. Los valores que se recogen en la tabla 7 permiten aceptar este nivel de invarianza. El índice de ajuste general (GFI= .951) y el error cuadrático medio de aproximación (RMSEA $=.037$ ) siguen aportando información convergente en esta dirección. Además, el criterio de información de Akaike $(A I C=138.099)$ y el índice comparativo de Bentler $(C F I=.973)$ no sufren grandes variaciones respecto al modelo anterior. Haciendo uso del criterio para la evaluación de los modelos anidados propuesto por Cheung y Rensvold (2002), quiénes sugieren que si el cálculo de la diferencia de los CFI de ambos modelos anidados disminuye en .01 o menos, se da por bueno el modelo restringido y por tanto el cumplimiento de la invarianza factorial; la diferencia entre CFIs obtenida .001 permite aceptar el modelo de invarianza métrica. Podemos concluir hasta ahora que las cargas factoriales son equivalentes en las dos muestras.

Una vez demostrada la invarianza métrica entre las muestras, pasamos a evaluar la equivalencia entre interceptos (invarianza factorial fuerte). Los índices (Tabla 7) muestran un buen ajuste de este modelo, tanto evaluado de modo independiente como analizándolo respecto a su anidamiento con el modelo de invarianza métrica. La diferencia entre los índices comparativos de Bentler es de .001; el índice de ajuste general es .972 y el error cuadrático medio de aproximación es .032. Aceptada la invarianza fuerte, los dos modelos evaluados son equivalentes respecto a los coeficientes factoriales y a los interceptos.

Tabla 7: Índices de bondad de ajuste de cada uno de los modelos puestos a prueba en la invarianza factorial; * $p<.01$.

\begin{tabular}{lccccccc}
\hline \multirow{2}{*}{ Modelo } & \multicolumn{7}{c}{ Índice de Ajuste } \\
\cline { 2 - 8 } & $\chi^{2}$ & gl & GFI & NFI & CFI & RMSEA & AIC \\
\hline Modelo sin restricciones & $53.070^{*}$ & 28 & .977 & .948 & .974 & .039 & 141.070 \\
Invarianza métrica & $58.099^{*}$ & 32 & .976 & .943 & .973 & .037 & 138.099 \\
Invarianza factorial fuerte & $66.943^{*}$ & 42 & .972 & .935 & .974 & .032 & 126.943 \\
\hline
\end{tabular}

\section{CONCLUSIONES}

De los resultados mostrados, de su análisis y de su discusión, y tomando en cuenta que el objetivo principal de este estudio fue el de examinar la estructura factorial y la medición de la invariancia de dicha estructura en alumnos de ingeniería, se pueden obtener las siguientes conclusiones: 1) El Análisis factorial exploratorio del instrumento autoeficacia en conductas de cuidado de la salud llevado a cabo en cada una de las submuestras estudiadas puso de manifiesto una estructura de cuatro factores: Alimentación, Adicciones, Salud Física e Hidratación; 2) los factores en ambas muestras evidenciaron una consistencia interna adecuada, particularmente si se considera el número reducido de ítems en cada uno de ellos; 3 ) el Análisis Factorial Confirmatorio indicó que el ajuste de los datos al modelo teórico de 8 ítems agrupados en cuatro factores es óptimo. Al mismo tiempo que los cuatro factores así obtenidos presentan en general saturaciones factoriales estandarizadas adecuadas. Por su parte los cuatro factores correlacionan entre sí de forma positiva y estadísticamente significativa lo cual muestra que a medida que aumenta la autoeficacia percibida en alguno de los factores, también aumenta en los otros dos; 4) Conjuntamente con todo lo antes dicho, los resultados del análisis de la invarianza factorial entre las submuestras; indican una alta congruencia entre pares de factores. Lo que sugiere la existencia de fuertes evidencias de la validación cruzada de la medida y por tanto de la estabilidad de la estructura, hasta que no se demuestre lo contrario. Futuras investigaciones deberían replicar estos hallazgos en muestras más amplias.

En síntesis, el análisis de las propiedades psicométricas, ha mostrado que una estructura tetrafactorial es viable y apropiada de acuerdo a los requisitos psicométricos establecidos cuando los informantes son los propios alumnos. La estructura de cuatro factores, atendiendo a criterios 
estadísticos y sustantivos, ha mostrado adecuados indicadores de ajuste, de fiabilidad y de validez. Sin embargo, consideramos que más estudios son necesarios con el fin de corroborar o refutar los datos obtenidos en la presente investigación.

\section{REFERENCIAS}

Abalo, J., Lévy, J., Rial, A., y Varela, J., Invarianza factorial con muestras múltiples. En J. Lévy (Ed.), Modelización con Estructuras de Covarianzas en Ciencias Sociales pp. 259-278. Netbiblo, Madrid (2006).

Arbuckle, J., AMOS users guide version 16.0 (2007).

Bandura, A., Exercise of personal agency throught the self-efficacy mechanism. In R. Schwarzer (Ed.), Self-efficacy: thought control of action, pp. 3-38, Hemisphere, Washington, DC, (1992).

Bandura, A., Self-efficacy: The exercise of Control, Freeman, New York (1997).

Blanco, H., Martínez, M., Zueck, M. A. y Gastélum, G., Análisis psicométrico de la escala autoeficacia en conductas académicas en universitarios de primer ingreso, Actualidades Investigativas en Educación, 11(3), 1-27 (2011).

Blanco, H. y otros siete autores, Editor para la construcción y aplicación de escalas por medio de una PC, Tecnociencia Chihuahua, 1(1), 55-59 (2007).

http://tecnociencia.uach.mx/numeros/v1n1/data/Editor_para_la_Construccion_y_Aplicacion_de_Es calas_por_medio_de_una_PC.pdf

Carbonero, M. A. y Merino, E., Autoeficacia y madurez vocacional, Psicothema, ISSN: 1886-144X (en línea), 16(2), 229-234, 2008. http://www.psicothema.com/pdf/1187.pdf. Acceso: 3 de agosto (2011).

Castañeda, S., Pineda, M. L., Gutierrez, E., Romero, N., Peñalosa, E., Construcción de instrumentos de estrategias de estudio, autorregulación y epistemología personal. Validación de constructo, Revista Mexicana de Psicología, 27(1), 77-85 (2010).

Cheung, G. W. y Rensvold, R. B., Evaluating goodness-of-fit indexes for testing measurement invariance, Structural Equation Modeling, 9, 233-255 (2002).

Costello, A. y Osborne, J., Best practices in exploratory factor analysis: four recommendations for getting the most from your analysis. Practical Assessment, Research and Evaluation, ISSN: 15317714, 10(7), 1-9, 2005. http://pareonline.net/pdf/v10n7.pdf. Acceso: 9 de Mayo (2011)

Elosua, P. y Zumbo, B., Coeficientes de fiabilidad para escalas de respuesta categórica ordenadas, Psicothema, ISSN: 1886-144X (en línea), 20(4), 896-901, 2008. http://www.psicothema.com/pdf/3572.pdf. Acceso: 3 de agosto (2011).

Gelabert, E. y otros cinco autores, Psychometric properties of the Spanish version of the Frost Multidimensional Perfectionism Scale in women, Psicothema, ISSN: 1886-144X (en línea), 23(1), 133-139, 2011. http://www.psicothema.com/pdf/3861.pdf. Acceso: 3 de agosto (2011).

Klein-Hessling, J., Lohaus, A. y Ball, J., Psychological predictors of health-related behaviour in children, Psychology, Health y Medicine, 10 (1) 31-43 (2005).

Leighton, J., Gokiert, R. y Cui, Y., Using Exploratory and Confirmatory Methods to Identify the Cognitive Dimensions in a Large-Scale Science Assessment, International Journal of Testing, 7(2), 141-189 (2007). 
León-Rubio, J. M., Cantero, F. J. y León-Pérez, J. M., Diferencias del rol desempeñado por la autoeficacia en el burnout percibido por el personal universitario en función de las condiciones de trabajo, Anales de Psicología, 27(2), 518-526. (2011).

Nunnally, J. y Bernstein, I., Teoría Psicométrica, McGraw-Hill, México (1995).

Olivari, C. y Urra, E., Autoeficacia y conductas de salud, Cienc. enferm. , ISSN: 0717-9553 (en línea), 13(1), 9-15 2007. http://www.scielo.cl/pdf/cienf/v13n1/art02.pdf. Acceso: 5 de agosto (2011).

Ornelas, M., Blanco, H., Gastélum, G. y Chávez, A., Autoeficacia percibida en conductas académicas en mujeres universitarias, Form. Univ., 5(2), en prensa (2012).

Ornelas, M., Blanco, H., Rodríguez, J. M. y Flores, F. J., Análisis Psicométrico de la Escala Autoeficacia en Conductas de Cuidado de la Salud Física en Universitarios de Primer Ingreso, Form. Univ., 4(6), (2011).

Pérez, E., Lescano, C., Zalazar, P., Furlám, L. y Martínez, M., Desarrollo y análisis psicométricos de un Inventario de Autoeficacia para Inteligencias Múltiples en Niños Argentinos. Psicoperspectivas, ISSN: 0718-6924 (en línea), $10 \quad$ (1), 169-189, 2011. http://www.psicoperspectivas.cl/index.php/psicoperspectivas/article/viewFile/141/129. Acceso: 3 de agosto (2011).

Raviolo, A., Ramirez, P., López, E. A. y Aguilar, A., Concepciones sobre el Conocimiento y los Modelos Científicos: Un Estudio Preliminar, Form. Univ., ISSN: 0718-5006 (en línea), 3(5), 29-36, 2010. http://www.scielo.cl/pdf/formuniv/v3n5/art05.pdf. Acceso: 3 de agosto (2011).

Sansinenea, E. y otros seis autores, Autoconcordancia y autoeficacia en los objetivos personales: ¿Cuál es su aportación al bienestar?, Anales de Psicología, 24(1), 121-128 (2008).

Thompson, B., Exploratory and Confirmatory Factor Analysis. Understanding concepts and applications, American Psychological Association, Washington, D C: (2004).

Vera, M., Salanova, M. y Martín-del-Río, B., Self-efficacy among university faculty: how to develop an adjusted scale, Anales de Psicología, 27(3), 800-807 (2011).

Wolters, C., Advancing achievement goal theory: using goal structures and goal orientations to predict students motivation, cognition and achievement, Journal of Educational Psychology, 96(2), 236-250 (2004). 
